\title{
Insights into the molecular mechanism of metal transport by NRAMP family transporters
}

\author{
S Ray ${ }^{1}, \mathrm{R}$ Gaudet ${ }^{2}$ \\ ${ }^{1}$ Harvard University, Brighton, MA, ${ }^{2}$ Professor, Cambridge, MA \\ shamayeeta ray@fas.harvard.edu
}

Transition metal ions like $\mathrm{Mn} 2+, \mathrm{Fe} 2+, \mathrm{Ni} 2+, \mathrm{Co} 2+, \mathrm{Cu} 2+$, and $\mathrm{Zn} 2+$ are essential nutrients that play a crucial role in various metabolic processes in all living cells. Excess or deficiency of these metal ions leads to harmful diseases including cancer, anemia and immune deficiencies. Cellular levels of essential metal micronutrients are thus tightly controlled and regulated. NRAMPs (natural resistance-associated macrophage proteins) are a class of transition metal transporters present in all domains of life that regulate the levels of these essential divalent metal ions within cells and prevent human disorders related to metal insufficiency (like anemia) or overload. In humans, there are two NRAMPs. NRAMP1 is expressed in macrophages and assists in immune response by preventing microbial access to $\mathrm{Mn} 2+$ and $\mathrm{Fe} 2+$ within pathogen-infected phagosomes. NRAMP2 is widely expressed at low levels in the endosomes of all nucleated cells, and also at the apical surface of epithelial cells in the intestinal tract, where it is responsible for uptake of non-heme Fe2+ from the diet. NRAMP-mediated metal ion transport typically involves co-transported protons. A few structures of bacterial NRAMPs are reported to date which reveal the overall fold (similar to LeuT permease) and provide a preliminary understanding of metal binding and proton co-transport. To obtain a better picture of metal ion selectivity, we obtained additional high-resolution crystal structures in multiple conformational states in apo and metal-bound forms of a bacterial NRAMP from Deinococcus radiodurans (DraNRAMP). To complement the structural data, we performed binding studies using ITC and proteoliposomebased transport assays to understand the thermodynamic parameters associated with metal binding and transport. Overall, the high-resolution structures of DraNRAMP provide a first look at the detailed coordination geometry for the bound metals in the transporter's metal-binding site with insights into metal selectivity determinants.

Acta Cryst. (2020). A76, a10 